Recepción: 20 / 12 / 2017

Aceptación: 15 / 02 / 2018

Publicación: 21 / 04 / 2018

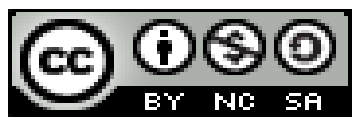

Ciencias Químicas

Artículo de Revisión

\title{
Evaluación de la contaminación físico-química de las aguas del estero salado, sector norte de la ciudad de Guayaquil-Ecuador-2017
}
Evaluation of physicochemical pollution of estero salado's water, north sector of Guayaquil city, Ecuador 2017

\section{Avaliação da contaminação físico-química das águas do estuário salgado, setor norte da cidade de Guayaquil-Equador-2017}

\begin{abstract}
Walter E. Mariscal-Santi ${ }^{\text {I }}$
walter.mariscals@ug.edu.ec

Frella S. Garcia-Larreta II

frella.garcia@hotmail.com

Raisa S. Mariscal-Garcia III

raisamariscal@hotmail.com

Andrea V. Cornejo-Ortega IV

cornejoandrea@gmail.com
\end{abstract}

Patricia M. Ortega-Ramirez V

patriortegar22@yahoo.com

Troski A. Montiel-Rivera VI

troskymontielrivera@gmail.com

Henry X. Ponce-Solórzano VII

henryxponce@gmail.com

Eduardo F. De La Torre-Quinonez VIII eduardofrancisco.delatorre@gmail.com

\section{Correspondencia: walter.mariscals@ug.edu.ec}

I Docente, Universidad de Guayaquil, Guayaquil, Ecuador.

II Docente, Universidad de Guayaquil, Guayaquil, Ecuador.

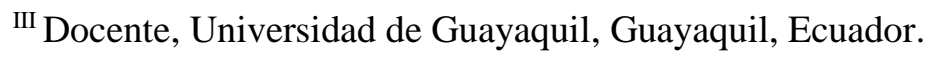

${ }^{\text {IV }}$ Docente, Universidad de Guayaquil, Guayaquil, Ecuador.

v Docente, Universidad de Guayaquil, Guayaquil, Ecuador.

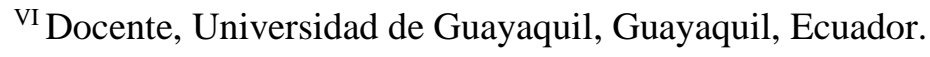

VII Docente, Universidad de Guayaquil, Guayaquil, Ecuador.

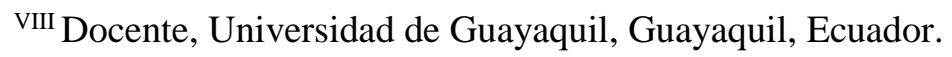


Walter E. Mariscal-Santi; Frella S. Garcia-Larreta; Raisa S. Mariscal-Garcia; Andrea V. Cornejo-Ortega; Patricia M. Ortega-Ramirez; Troski A. Montiel-Rivera; Henry X. Ponce-Solórzano; Eduardo F. De La Torre-Quinonez

\section{Resumen}

La investigación se ejecutó en el sector norte del Estero Salado de la ciudad de Guayaquil, en los tramos comprendidos del puente de Miraflores, pasando por el puente Albán Borja, hasta el puente de Urdesa, durante el periodo de Junio a noviembre del 2017, estableciéndose un sistema de 9 puntos de muestreo, tres puntos por cada puente (orilla, centro y extremo), manteniendo un monitoreo de tres fechas programadas. Los objetivos fueron determinar la contaminación físicoquímica de las aguas del estero salado de los puentes antes indicados e identificar el tramo de mayor contaminación.

Las principales actividades que tributan contaminantes al estero de Urdesa y Miraflores son 573 industrias - ya identificadas por el Ministerio del Ambiente - mediante el vertido de sus aguas residuales por canales no regulados, desechos sólidos y domésticos de la población, descargas de escorrentías, tuberías de desagües clandestinas. Finalmente se determinó que el sector del puente de la ciudadela Miraflores es el que presenta mayor contaminación, desde el punto de vista de carga orgánica e inorgánica.

Palabras clave: Contaminación físico-química de aguas, Estero Salado, norte de Guayaquil Ecuador. 


\section{Abstract}

The investigation was carried out in the northern part of Estero Salado's of Guayaquil city, in the Miraflores bridge section, over the bridge Albán Borja, until Urdesa bridge during the period from June to November 2017, establishing a system of 9 sampling points, three points for each bridge (edge, middle and end), keeping monitoring three scheduled dates. The objectives were to determine the physical-chemical pollution salty waters of the estuary of the bridges listed above and identify the most polluted stretch.

The main activities that confer Urdesa's estuary pollutants and Miraflores are 573 industries - as identified by the Ministry of Environment - by pouring their waste water on unregulated channels, domestic and population solid waste, runoff downloads, underground pipes drains. Finally determined that the sector of Miraflores's citadel bridge is the most contaminated point from organic and inorganic load.

Keywords: Pollution physicochemical of water, Salado Estuary, north of Guayaquil - Ecuador. 


\section{Introducción.}

Desde épocas remotas el hombre aprendió a sacar provecho del mar, incluso hasta el día de hoy, principalmente a lo largo de las franjas costeras y de los esteros, realizando de esta forma la mayoría de sus actividades en este entorno, aprovechándose de sus recursos (flora y fauna).

Dentro de su fauna tenemos peces, crustáceos, moluscos, etc. que le dotan de alimentos ricos en proteínas y grasas, pero también se aprovecha de recursos inertes tales como: minerales, sal común, etc. (Orozco 2005)

El ramal del Estero Salado ubicado en el sector Norte de la ciudad de Guayaquil (urbanizaciones Urdesa y Miraflores), es un brazo de mar y como tal está sujeto a las acciones de flujo y reflujo de las mareas por lo tanto la introducción del agua nueva del mar abierto al estero es muy limitada, este comportamiento resulta perjudicial desde el punto de vista de la regeneración de la calidad de sus aguas.

El ritmo de contaminación del Estero Salado sector norte, se ha visto incrementado por el desequilibrio existente entre el crecimiento poblacional descontrolado, el incremento de industrias, locales de entretenimiento, restaurantes, hotelería, peluquerías, lavanderías, llanteras y una gran variedad de comercios formales e informales con los servicios de infraestructura muy especialmente al alcantarillado sanitario que han colapsado, provocando el grave problema de contaminación del estero, tanto de materia orgánica como inorgánica, al punto que los ciudadanos de ese sector están acostumbrados al mal olor del ambiente, causado en gran medida por la descomposición de los desechos arrojados al estuario; la supervivencia de la flora y fauna, exige que el agua y sus sedimentos esté libre de sustancias tóxicas y que su concentración de oxígeno sea adecuada, lo cual no sucede en los lugares en que se realizó el muestreo, ya que la concentración de oxígeno en agua 
Evaluación de la contaminación físico-química de las aguas del estero salado, sector norte de la ciudad de Guayaquil-Ecuador-

es muy cercana al cero; la escasez de oxígeno hizo que el proceso de depuración natural de las aguas del Estero Salado en el sector norte, no se pueda efectuar, acentuándose de esta forma el problema de contaminación. Además análisis realizados ( Valencia 2001 ) determinaron que existe gran concentración de metales nocivos para la salud tales como mercurio, cadmio y plomo, por otro lado existe una elevada concentración de aceites lo cual impide que penetre la luz y el oxígeno necesarios para los diferentes procesos que se deben dar en las aguas del Estero Salado sector norte debido a la escasez de oxígeno, la Demanda Bioquímica de Oxígeno ( DBO5 ) es alta, consecuencia de la acumulación de la población microbiana, excesiva presencia de residuos desagradables.

No se han respetado las Leyes Municipales y Ambientales que prohíben arrojar efluentes contaminantes y hubo poca vigilancia en el cumplimiento de estas disposiciones legales, esto ha ocasionado procesos de eutrofización, desestabilizando el ecosistema existente aún más, tratándose del manglar y otros tipos de flora que son ecosistemas frágiles, hábitat natural de especies faunísticas, que constituyen atractivos turísticos y reguladores ambientales de este sector estuarino de la ciudad de Guayaquil. (Medina, J. Monserrate, L. P. Calle)

Ante esta ineludible realidad, el presente trabajo de investigación, se ha propuesto como uno de sus principales objetivos: "determinar la contaminación físico-química de las aguas del Estero Salado, sector norte de la ciudad de Guayaquil- Ecuador, identificando la zona de mayor contaminación dentro del mismo, que abarca las ciudadelas Miraflores y Urdesa"

La importancia básica de la presente investigación, radica en que nos proporcionaría un estudio completo de los efectos de la calidad del agua del Estero Salado, sector norte de la ciudad de Guayaquil, registrados en el año 2013, con un enfoque investigativo, descriptivo, explicativo, metodológico y cuantitativo; que se constituya en una propuesta a considerar en las diferentes acciones de remediación ambiental de este importante tramo del estero salado por parte de las 
Walter E. Mariscal-Santi; Frella S. Garcia-Larreta; Raisa S. Mariscal-Garcia; Andrea V. Cornejo-Ortega; Patricia M. Ortega-Ramirez; Troski A. Montiel-Rivera; Henry X. Ponce-Solórzano; Eduardo F. De La Torre-Quinonez

entidades gubernamentales, municipales y ambientales, llegando a uno de los componentes más importantes de esta tarea, como es la educación ambiental y social, por medio del cual se debe trabajar en la sensibilización y concienciación de la población para promover la participación y el empoderamiento de la comunidad vecina del estero, que permita aplicar campañas de concienciación ambiental y asegurar la mejor gestión de los desechos sólidos; la generación de políticas motivacionales, fiscales, participativas, reconociendo en la gestión de la comunidad, su potencial y desarrollo en este tema de investigación.

\section{Resultados y discusión.}

Parámetros fisicos

a) Potencial de Hidrogeno (pH)

El Potencial de hidrógeno, para el mes de Agosto, presentó un promedio de 7.31 UpH, con una desviación estándar de 0.478 g, siendo el ph más alto el registrado en el puente Miraflores, cuyo valor fue de 7.36 $\mathrm{UpH}$. lo que se considera un valor normal dentro del rango establecidos por el TULSMA que es de (6.5 a 9.5)

El valor del pH en el puente Albán Borja, fue de $7.33 \mathrm{UpH}$, el $\mathrm{pH}$ del puente de Urdesa fue de 7.25 UpH, lo que se considera un valor normal dentro del rango establecidos por el TULSMA que es de (6.5 a 9.5). Para las tres estaciones analizadas los resultados análisis de $\mathrm{pH}$ mostraron que la acidez va descendiendo según nos movemos del puente UNO, hasta adentro del puente TRES. Siendo la estación del puente uno en la que el pH fue mayor de 7.36. Estas ligeras variaciones pueden estar relacionadas por la gran cantidad de materia orgánica que entra al sistema por la disposición de aguas servidas por alrededor de más de un centenar de residencias entre los puentes 
Evaluación de la contaminación físico-química de las aguas del estero salado, sector norte de la ciudad de Guayaquil-Ecuador-

uno al tres principalmente, y otras fuentes de materia o sustancias orgánicas como hojas, ramas de mangle y aceite $\mathrm{u}$ otros hidrocarburos en las aguas de escorrentías.

El potencial de hidrógeno para el mes de Octubre presentó un promedio de 7.267 UpH, con una desviación estándar de 0.057 , siendo el pH más alto la registrada en el puente Miraflores, cuyo valor fue de $7.33 \mathrm{UpH}$.

El Potencial de hidrógeno, para el mes de diciembre, presentó un promedio de 7.291 UpH, con una desviación estándar de 0.055 , siendo el pH más alto la registrada en el puente Miraflores, cuyo valor fue de $7.35 \mathrm{UpH}$, lo que se considera un valor normal dentro del rango establecidos por el TULSMA que es de (6.5 a 9.5)

\section{b).- Temperatura}

La temperatura influye en la sensibilidad de las sales presentes en el agua, los gases y el pH. La temperatura superficial del agua para el mes de agosto, presentó un promedio de $25.3^{\circ} \mathrm{C}$, con una desviación estándar de 0.433 , siendo la temperatura más baja la registrada en el puente Miraflores con valores de $24.8^{\circ} \mathrm{C}$, en tanto que las temperaturas registrada en los puentes de Urdesa y Albán Borja fueron similares de $25.3{ }^{\circ} \mathrm{C}$ y $25.8^{\circ} \mathrm{C}$.Estos valores son normales, y están en los límites que establece el TULSMA y porque en estos meses la temperatura máxima a la que llega la estación climática fue de $28^{\circ} \mathrm{C}$.

Para el mes octubre, presentó un promedio de $23.37^{\circ} \mathrm{C}$, con una desviación estándar de 0.43 , siendo la temperatura más baja la registrada en el puente Miraflores con valores de $22.8^{\circ} \mathrm{C}$, en tanto que las temperaturas registrada en los puentes de Urdesa y Albán Borja fueron similares de $23.7^{\circ} \mathrm{C}$ y $23.6^{\circ} \mathrm{C}$. Estos valores son normales, y están en los límites que establece el TULSMA y porque en estos meses la temperatura máxima a la que llega la estación climática fue de $28^{\circ} \mathrm{C}$. 
Walter E. Mariscal-Santi; Frella S. Garcia-Larreta; Raisa S. Mariscal-Garcia; Andrea V. Cornejo-Ortega; Patricia M. Ortega-Ramirez; Troski A. Montiel-Rivera; Henry X. Ponce-Solórzano; Eduardo F. De La Torre-Quinonez

Para el mes de diciembre, presentó un promedio de $24.37^{\circ} \mathrm{C}$, con una desviación estándar de 0.44, siendo la temperatura más baja la registrada en el puente Miraflores con valores de $23.1^{\circ} \mathrm{C}$, en tanto que las temperaturas registrada en los puentes de Urdesa y Albán Borja fueron similares de $24.5^{\circ} \mathrm{C}$ y $25.5^{\circ} \mathrm{C}$.Estos valores son normales, y están en los límites que establece el TULSMA y porque en estos meses la temperatura máxima a la que llega la estación climática fue de $28^{\circ} \mathrm{C}$.

\section{C.- Turbidez}

La turbidez de un agua superficial se debe principalmente a la presencia de los sólidos en suspensión (microorganismos, arcillas, limos, fitoplancton, etc.), para el mes de agosto, los valores promedios de sólidos disueltos totales fueron: puente de Miraflores 172 NTU, puente Albán Borja 169.5 NTU, puente de Urdesa 119.02 NTU, lo que hace un promedio general de 153.51 NTU.

Para el mes de octubre, los valores promedios encontrados en el tramo comprendido entre el Puente Miraflores al Puente de Urdesa fueron los siguientes:170 NTU, 168 NTU y 118 NTU, lo que hace un promedio de 152 NTU, La diferencia entre los valores mencionados se debe fundamentalmente al movimiento del agua.

Para el mes de Diciembre, los valores promedios de sólidos disueltos totales fueron: puente de Miraflores 174 NTU, puente Albán Borja 167 NTU, puente de Urdesa 117 NTU, lo que hace un promedio de 152.7 NTU. Estos valores están muy por encima de los estándares establecidos por las TULSMA.

d). - Sólidos disueltos totales

La determinación de sólidos disueltos totales mide específicamente el total de residuos sólidos filtrables (sales y residuos orgánicos). Los sólidos disueltos pueden afectar adversamente la calidad de un cuerpo de agua o un efluente de varias formas. Los análisis de sólidos disueltos son 
Evaluación de la contaminación físico-química de las aguas del estero salado, sector norte de la ciudad de Guayaquil-Ecuador-

también importantes como indicadores de la efectividad de procesos de tratamiento biológico y físico de aguas de esteros. Para el mes de agosto, los valores promedios de sólidos disueltos totales fueron: puente de Miraflores 29 mg/L, puente Albán Borja $145.1 \mathrm{mg} / \mathrm{L}$, puente de Urdesa $174 \mathrm{mg} / \mathrm{L}$.

Para el mes de octubre, los valores promedio en el puente Miraflores fue de $29.45 \mathrm{mg} / \mathrm{L}$, en el puente de Albán Borja de145.7 mg/L y el puente de Urdesa fue de174.5 mg/L, lo que nos indica que existe alta contaminación por efluentes de desperdicios domésticos, aguas negras, desechos industriales y escorrentías.

Para el mes de Diciembre, los valores promedios fueron: puente de Miraflores $29.3 \mathrm{mg} / \mathrm{L}$, puente Albán Borja $145.6 \mathrm{mg} / \mathrm{L}$, puente de Urdesa $174.8 \mathrm{mg} / \mathrm{L}$.

\section{e).- Salinidad}

Es la determinación de la masa de sales disueltas en una masa dada de solución, para determinarla se utilizó métodos indirectos como la conductividad, la densidad o el índice de refracción. Para el mes de agosto, los valores promedios de salinidad fueron: puente de Miraflores 562 mg/L, puente Albán Borja 34 mg/L, puente de Urdesa 16.8 mg/L.

Para el mes de octubre, los valores promedios de salinidad fueron: puente de Miraflores 561 mg/L, puente Albán Borja $31.5 \mathrm{mg} / \mathrm{L}$, puente de Urdesa $16.5 \mathrm{mg} / \mathrm{L}$.

Para el mes de diciembre, los valores promedios de salinidad fueron: puente de Miraflores $565 \mathrm{mg} / \mathrm{L}$, puente Albán Borja $35.5 \mathrm{mg} / \mathrm{L}$, puente de Urdesa $16.8 \mathrm{mg} / \mathrm{L}$. Estos valores están muy por encima de los estándares establecidos por las TULSMA. 


\section{Parametros químicos}

\section{a) Oxigeno Disueltos}

Oxígeno Disuelto (OD), es el oxígeno libre que se encuentra en el agua, elemento vital para la supervivencia de todas las formas de vida acuática. La solubilidad del oxígeno depende de la concentración de sales disueltas y sobre todo de la temperatura y presión atmosférica. En el mes de Agosto los valores reportados por el laboratorio fueron de $0.87 \mathrm{mg} / \mathrm{L}$, puente Miraflores, 1.20 mg/L, puente Albán Borja y 2.04 mg/L, puente Urdesa.

Para el mes de octubre los valores promedios de oxígeno disuelto determinados dentro del sector de investigación fueron de $0.85,1.15$, y 2,01 mg/L respectivamente; además se determinó un bajo nivel de oxígeno disuelto en las TRES estaciones variaron, dichos rangos demuestran un patrón ascendente según la estaciones de muestreo. Coincidiendo, los valores más bajos con niveles hipóxicos donde no se puede mantener organismos vivos, localizados en la zona de la ciudadela Miraflores, lugar de mayor descarga de aguas servidas sin tratamiento.

En el mes de diciembre los valores reportados por el laboratorio fueron de $0.84 \mathrm{mg} / \mathrm{L}$, puente Miraflores, $1.18 \mathrm{mg} / \mathrm{L}$, puente Albán Borja y $2.05 \mathrm{mg} / \mathrm{L}$, puente Urdesa. Estos valores están muy por debajo de los estándares establecidos por las TULSMA.

\section{b) Demanda Bioquímica de Oxígeno}

La determinación de oxígeno para oxidar la materia orgánica de una agua residual, se usa cuando es necesario la inhibición de la nitrificación de una población microbiana, por ello se define a la demanda bioquímica de oxígeno, DBO5, como la cantidad de oxígeno disuelto requerido por los microorganismos aerobios para la degradación de la materia orgánica. En el mes de agosto los valores reportados por el laboratorio fueron de $17 \mathrm{mg} / \mathrm{L}$, puente Miraflores, $15 \mathrm{mg} / \mathrm{L}$, puente Albán 
Evaluación de la contaminación físico-química de las aguas del estero salado, sector norte de la ciudad de Guayaquil-Ecuador-

Borja y $10 \mathrm{mg} / \mathrm{L}$, puente Urdesa. La demanda bioquímica de oxígeno, presentó además un promedio de $14 \mathrm{mg} / \mathrm{L}$, con una desviación estándar de 3.12, siendo la DBO5 más alta, la registrada en el puente Miraflores.

En el mes de octubre los valores promedios reportados por el laboratorio fueron de $18 \mathrm{mg} / \mathrm{L}$, puente Miraflores, $15 \mathrm{mg} / \mathrm{L}$, puente Albán Borja y $11 \mathrm{mg} / \mathrm{L}$, puente Urdesa. Los valores encontrados reflejan un incremento considerablemente superior; esto se debe principalmente a la contaminación de las aguas por introducción de materia orgánica.

En el mes de diciembre los valores reportados por el laboratorio fueron de $17.5 \mathrm{mg} / \mathrm{L}$, puente Miraflores, $14.5 \mathrm{mg} / \mathrm{L}$, puente Albán Borja y $10.5 \mathrm{mg} / \mathrm{L}$, puente Urdesa. La demanda bioquímica de oxígeno, presentó además un promedio de $14.166 \mathrm{mg} / \mathrm{L}$, con una desviación estándar de 3.0413, siendo la DBO5 más alta, la registrada en el puente Miraflores. Estos valores están muy por encima de los estándares establecidos por las TULSMA.

\section{c.- Demanda Química de Oxígeno}

La DQO, es otra manera de determinar la materia orgánica biodegradable y no biodegradable presente en el agua y/o los sedimentos; desgraciadamente una de las interferencias más negativas y la más importante es la presencia de cloruros en la muestra. Esto hace que a valores de cloruros mayores a $2000 \mathrm{mg} / \mathrm{L}$ cualquier resultado es irreal.

Dentro del caso que nos ocupa no es la excepción por ello los valores encontrados podrían ser comparados con ciertos valores de aguas residuales industriales altamente contaminadas, pero realmente no es así, es la alta salinidad encontrada la que causa estos resultados.

En el mes de agosto los valores reportados por el laboratorio fueron de $154 \mathrm{mg} / \mathrm{L}$, puente Miraflores, $61 \mathrm{mg} / \mathrm{L}$, puente Albán Borja y $8.5 \mathrm{mg} / \mathrm{L}$, puente Urdesa. La demanda química de 
Walter E. Mariscal-Santi; Frella S. Garcia-Larreta; Raisa S. Mariscal-Garcia; Andrea V. Cornejo-Ortega; Patricia M. Ortega-Ramirez; Troski A. Montiel-Rivera; Henry X. Ponce-Solórzano; Eduardo F. De La Torre-Quinonez

oxígeno, presentó además un promedio de 74.5 mg/L, con una desviación estándar de 63.761, siendo la DQO más alta la registrada en el puente Miraflores.

En el mes de octubre los valores reportados por el laboratorio fueron de $155 \mathrm{mg} / \mathrm{L}$, puente Miraflores, $60 \mathrm{mg} / \mathrm{L}$, puente Albán Borja y $9 \mathrm{mg} / \mathrm{L}$, puente Urdesa. La demanda química de oxígeno, presentó además un promedio de $74.68 \mathrm{mg} / \mathrm{L}$, con una desviación estándar de 64.15, siendo la DQO más alta la registrada en el puente Miraflores.

En el mes de diciembre los valores reportados por el laboratorio fueron de $156 \mathrm{mg} / \mathrm{L}$, puente Miraflores, $63 \mathrm{mg} / \mathrm{L}$, puente Albán Borja y $8.5 \mathrm{mg} / \mathrm{L}$, puente Urdesa. La demanda química de oxígeno, presentó además un promedio de $75.83 \mathrm{mg} / \mathrm{L}$, con una desviación estándar de 64.6, siendo la DQO más alta la registrada en el puente Miraflores Estos valores están muy por encima de los estándares establecidos por las TULSMA.

c) Nitratos

En el análisis de nitratos en el agua, en el mes de agosto se determinó que la estación de muestreo del puente Miraflores tiene el valor promedio más bajo de(0.035 mg/L), la estación puente Albán Borja un valor promedio de $(0.21 \mathrm{mg} / \mathrm{L})$ y la estación del puente de Urdesa un valor promedio de $(0.30 \mathrm{mg} / \mathrm{L})$, como se conoce el Nitrato es una forma de nitrógeno que toda flora necesita para crecer y cuando se vierten residuos industriales con fertilizantes en abundancia y sin tratamiento previo los nitratos pueden contaminar las aguas del estero. Se establece el valor para el Nitrato de $<5 \mathrm{mg} / \mathrm{L}$, como valor máximo de concentración.

En el mes de octubre se determinó que la estación de muestreo del puente Miraflores tiene el valor promedio más bajo de $(0.038 \mathrm{mg} / \mathrm{L})$, la estación puente Albán Borja un valor promedio de (0.22 mg/L) y la estación del puente de Urdesa un valor promedio de $(0.32 \mathrm{mg} / \mathrm{L})$, 
Evaluación de la contaminación físico-química de las aguas del estero salado, sector norte de la ciudad de Guayaquil-Ecuador-

En el mes de diciembre se determinó que la estación de muestreo del puente Miraflores tiene el valor promedio más bajo de $(0.034 \mathrm{mg} / \mathrm{L})$, la estación puente Albán Borja un valor promedio de $(0.23 \mathrm{mg} / \mathrm{L})$ y la estación del puente de Urdesa un valor promedio de $(0.35 \mathrm{mg} / \mathrm{L})$.

d) Fosfatos

En el análisis de fosfatos en el agua, realizado en el mes de agosto, se determinó que la estación de muestreo del puente Miraflores tiene el valor promedio más bajo de( $0.43 \mathrm{mg} / \mathrm{L}), \mathrm{la}$ estación puente Albán Borja un valor promedio de $(0.81 \mathrm{mg} / \mathrm{L})$ y la estación del puente de Urdesa un valor promedio de $(1 \mathrm{mg} / \mathrm{L})$, lo que se considera un valor muy alto dentro del rango establecidos que es de $(0.1$ a $0.2 \mathrm{mg} / \mathrm{L})$, cabe anotar que los fosfatos son nutrientes de las plantas y conducen al crecimiento de algas en las aguas superficiales. Dependiendo de la concentración de fosfato existente en el agua, puede producirse la eutrofización. Hay que recordar que tan solo $1 \mathrm{~g}$ de fosfatofosforo (PO4-P) provoca el crecimiento de hasta $100 \mathrm{~g}$ de algas. Cuando estas algas mueren, los procesos de descomposición dan como resultado una demanda de oxigeno de alrededor de $150 \mathrm{~g}$

En el mes de octubre se determinó que la estación de muestreo del puente Miraflores tiene el valor promedio más bajo de $(0.46 \mathrm{mg} / \mathrm{L})$, la estación puente Albán Borja un valor promedio de $(0.81$ $\mathrm{mg} / \mathrm{L})$ y la estación del puente de Urdesa un valor promedio de $(1.01 \mathrm{mg} / \mathrm{L})$, lo que se considera un valor muy alto dentro del rango establecidos que es de $(0.1$ a $0.2 \mathrm{mg} / \mathrm{L})$

En el mes de diciembre, se determinó que la estación de muestreo del puente Miraflores tiene el valor promedio más bajo de $(0.47 \mathrm{mg} / \mathrm{L})$, la estación puente Albán Borja un valor promedio de (0.84 mg/L) y la estación del puente de Urdesa un valor promedio de $(1.06 \mathrm{mg} / \mathrm{L})$, lo que se considera un valor muy alto dentro del rango establecidos que es de $(0.1$ a $0.2 \mathrm{mg} / \mathrm{L})$, 
Walter E. Mariscal-Santi; Frella S. Garcia-Larreta; Raisa S. Mariscal-Garcia; Andrea V. Cornejo-Ortega; Patricia M. Ortega-Ramirez; Troski A. Montiel-Rivera; Henry X. Ponce-Solórzano; Eduardo F. De La Torre-Quinonez

\section{Bibliografía.}

Acosta A. Zapata A.G., Fagua. Técnicas de campo en Ambientes tropicales. Manual para el Monitoreo en ecosistemas acuáticos. Bogotá Pontificia Universidad Javeriana.

APHA. 1998, Standard Methods for the Examination of Water and Wastewater.

Atlas, Ronald M.R. Bartha: Ecología Microbiana y Microbiología Ambiental. Trad R. Guerrero 4 ed Madrid. Prentice Hall.

Ayarza, W., Coello, S., Chalén, N., Garcés, P., García, L., García, M.L., Omarza, F., Pérez, E., Pesantes, F., Solórzano, L. 1993 «Estudios Geobioquímicos de la Sección Urbana del Estero Salado.» Revista de Ciencias del Mar y Limnología. Guayaquil-Ecuador.

CAAM 1996, Desarrollo y Problemática Ambiental del área del Golfo de Guayaquil-Ecuador.

Carvajal, R., M. Jiménez, y P. Iturralde, 2006. Zonificación de la Reserva de Producción de Fauna Manglares El Salado. Editado por Fundación Natura. Guayaquil-Ecuador.

Chalén, N. 1986. Mercurio en el estuario interior del Golfo de Guayaquil incluyendo el Estero Salado, Rio Guayas y los cursos inferiores de los Ríos Daule y Babahoyo, Guayaquil Instituto Nacional de Pesca-Ecuador.

Constitución de la República del Ecuador. Montecristi - Manabí, 25 de julio de 2008.

DePauw, N. 2007. Biological monitorig and assessment of surface water quality. Curso de monitoreo de la calidad de aguas. Escuela Superior Politécnica del Litoral, Guayaquil-Ecuador.

Downes, B. Fairweather, P. Kenough, M. and Mapstone. B. 2002. Monitoring ecological impacts, concepts and practice in flowing waters Cambridge University Press- Cambridge-Inglaterra.

Eginseco Cia. 2001. Diagnóstico y Plan Ambiental del Terminal Fuel Oil de Petrocomercial en la ciudad de Guayaquil-Ecuador.

Empresa Municipal de Alcantarillado de Guayaquil (EMAG), 1980. Plan de Recuperación del Estero Salado primera parte. Guayaquil -Ecuador.

Enkerlin. 1997. Ciencia ambiente y desarrollo sostenible. México (MX): Thonsom Editores.

EPA. 2001. Nutrient Criteria Technical Guidance Manual. Estuarine and Coastal Marine Waters. United States Environmental Protection Agency Office of Water. Disponible -USA.

Estrella, T. 2000. Uso del Recurso Agua y Manglares en el Estero de Puerto Hondo. provincia del Guayas, Ecuador. tesis IV Maestria en conservación y gestión del medio natural: Integración de sistemas naturales y humanos. Guayaquil-Ecuador.

FAO 1981. Manual of Methods in aquatic environment research. Part 8, Ecological assessment of pollution effects (Guidelines for the FAO (GFCM)/UNEP Joint Coordinated Project on Pollution in the Mediterranean) Rome-Italy.

Fernández, C. 2005. Evaluación de Impacto Ambiental del Proyecto Centro Recreativo Balneario Puerto Hondo Parroquia tarqui, Guayaquil Fundación Guayaquil Siglo XXI-Ecuador. 
Glynn. 1999. Ingeniería ambiental. México (MX): Editorial Prentice Hall.

Harrison. 1999. Entendiendo nuestro ambiente: Una introducción a la química ambiental y la contaminación. España (ES): Editorial Acribia.

Hartman, 1996, Atlas of The Ssdentariate Polychaetous Annelids From California, Allan Hancock Foundation. Los Ángeles, California, EEUU.

Hidroestudios, 2001: Plan Integral de Recuperación el Estero Salado Fase 1, Guayaquil, Estudios de impacto Ambiental de los desvíos temporales de los tramos A y B del Estero Salado. GuayaquilEcuador.

Holland, A.F., y otros, 2004. Linkages between Tidal Creek Ecosystems and the Landscape and Demographic Attributes of their Watersheds. Journal Experimental Marine Biology and Ecology. USA.

Holland, A.F.; Sanger, D.M.; Gawle, C. P.; Lerberg, S.B.; Santiago, M.S.; Riekerk, G.H.M.; Zimmerman, L.E.; Scott, G.E. 2004. «Journal Experimental Marine Biology and Ecology. » Linkages between Tidal Creek Ecosystems and the Landscape and Demographic Attributes of their Watersheds. USA.

Instituto Nacional de Estadística y Censos (INEC). Censo de población y vivienda, 2010 .Ecuador.

Lahmeyer-Cimentaciones, 2000. «Estudios de Prefactibilidad, Factibilidad y Selección de la Mejor Alternativa del PIRES.» Parte II, Informe Final, Preparado para la Muy Ilustre Municipalidad de Guayaquil. Guayaquil - Ecuador.

LEY DE AGUAS.- República del Ecuador.- 20 de Mayo de 2004.

McGraw-Hill. 1990. Diccionario de Química. Tomo II. McGraw-Hill/Interamericana de México.

Manahan. 2007. Introducción a La química ambiental. México (MX): Editorial Reverte UNAM.

Medina, J. Monserrate, L. P. Calle. Evaluación de las condiciones químicas, físicas y biológicas del Estero Salado en el sector dentro de la ciudad de Guayaquil. Escuela Superior politécnica del Litoral. Guayaquil-Ecuador.

Ministerio del Ambiente. 2002. Texto Unificado de Legislación Ambiental Secundaria. edición Especial. Corporación de estudios y Publicaciones. Quito-Ecuador.

M.I. Municipalidad de Guayaquil, 2007. «Plan de Manejo Reserva de Producción Faunística Manglares el Salado.» Guayaquil-Ecuador.

Montaño, M. 2007. Estudio de la calidad del ambiente Estero Santa Ana-Salado frente a Trinipuerto. Auditoría Ambiental de cumplimiento. Transpuerto S.A. Dirección de Medio Ambiente del Municipio de Guayaquil-Ecuador.

Mora, E., Reinoso, B. 1981. «Investigaciones Preliminares sobre el estado actual de las poblaciones de ostiones en tres zonas del Estuario Interior del Golfo de Guayaquil.» Revista de Ciencias del Mar y Limnolo- Guayaquil-Ecuador

Murgel M. 1984. Linmología sanitaria estudio de la polución de aguas continentales. Estados Unidos (USA): Organización de Estados Americanos. 
Walter E. Mariscal-Santi; Frella S. Garcia-Larreta; Raisa S. Mariscal-Garcia; Andrea V. Cornejo-Ortega; Patricia M.

Ortega-Ramirez; Troski A. Montiel-Rivera; Henry X. Ponce-Solórzano; Eduardo F. De La Torre-Quinonez

Murray, S. D. A. Simpong and J. Santoro 1973. Circulation and Salinity distribution in the Rio Guayas. Estuary Ecuador. Coastal Estudies, L.S.U. USA.

Orozco et al. 2005. Contaminación ambiental - una visión desde la química. España (ES): Thompson Editores.

Pesantes, F.E. Pérez 1993. Estudio comparativo de las condiciones Físicas y Químicas de las aguas costeras ecuatorianas. 1982-1983. Revista Ciencias del Mar. Linmología.- Guayaquil-Ecuador.

Perdomo C, Casanova O, \& Ciganada V. 2001. Contaminación de aguas subterráneas con nitratos y coniformes en el litoral sudoeste del Uruguay. Agrociencia vol. $\mathrm{N}^{\circ} 1$.

Primer Simposio de Biodiversidad Marina y Costera de Latinoamérica y el Caribe y Segundo Simposio Nacional de Biodiversidad Marina y Costera. 2010. Diferencias en distribución y abundancia de macro invertebrados bentónicos en sectores del Estero Salado con diferente grado de influencia antropogénica. Manta - Ecuador.

Rivera, J., 2007. Reserva de Producción de Fauna Manglares el Salado. Quito 1- Ecuador.

Ridgely and Greenfield (2001) the Birds of Ecuador. Especies de rango restringido, listadas para la región de los Andes del sur.

Rodier, J. 1981. Análisis de las Aguas. Aguas naturales, aguas residuales y agua de mar. BarcelonaEspaña.: Omega.

Ruiz et al. 2006. Determinación de la demanda química de oxígeno y su importancia en la ingeniería ambiental. En 2o foro académico nacional de Ingenierías y Arquitectura- México.

Sawyer, C. McCarthy, P. and Parkin, G. 2000. Química para la Ingeniería Ambiental. Tracd McGarwHill. 4 ed. Bogotá. Co. Interamericana.

Soanez. 1999. Ingeniería del Medio Ambiente - Aplicada al medio ambiente natural continental. España (ES): Ediciones Mundi-Prensa.

Solórzano, L., Viteri, G., 1993. «Investigación Química del Estero Salado.» Revista de Ciencias del Mar y Limnología III.- Guayaquil-Ecuador.

Spiro \& Stigliani. 2004. Química medioambiental. España (ES): Editorial Pearson Educación S.A.

Tyler M. 2004. Ciencia Ambiental -Preservemos la tierra. México (MX): Editorial Thomsom.

Universidad de Guayaquil. 2008. Evaluación del nivel de contaminación del manglar y aguas del Estero Salado circundante a la Estación de Transferencia Tres Bocas de Petrocomercial Sur, con miras a establecer un plan de manejo Ambiental y de mitigación, Dirección de Investigaciones y Proyectos Académicos (DIPA) Facultad de Ingeniería Química, Departamento de Petróleo y Petroquímica..- Guayaquil-Ecuador.

Valencia, Manuel, 2001. Contaminantes del Estero Salado. Técnico, Guayaquil- Ecuador.

Vives De Andréis, José Benito, 2007. Manual de Técnicas Analíticas para la determinación de parámetros fisicoquímicos y contaminantes marinos (aguas, sedimentos y organismos). Programa Calidad Ambiental Marina - CAM. Ecuador 
Wiessman \& Hammer. 2005. Water supply and pollution control. Estados Unidos (USA): Pearson Prentice Hall Edition.

Wong and Wong, 2007. Estudio de Impacto Ambiental fase de operación, Terminal Portuario Internacional Puerto Hondo. Guayaquil-Ecuador.

Zambrano López, Nelson. 2007. Estudio de impacto ambiental del parque. recopilado por M. I. Municipalidad de Guayaquil. Ecuador.

Zambrano, N. 2007. Programa de Manejo de Recursos Costeros. Plan de Manejo del Bosque Protector Estero Salado. Guayaquil Ecuador. 